\title{
Complex Formation between Bovine Serum Albumin and Strong Polyelectrolytes: Effect of Polymer Charge Density
}

\author{
Kevin W. Mattison, Paul L. Dubin,* and Isabelle J. Brittain \\ Department of Chemistry, Indiana University-Purdue University, Indianapolis, Indiana 46202
}

Received: December 10, 1997; In Final Form: February 25, 1998

\begin{abstract}
Light scattering and $\mathrm{pH}$ titration were used to examine the binding of bovine serum albumin (BSA) to poly(diallyldimethylammonium chloride) (PDADMAC), poly(acrylamidomethylpropyl sulfonate) (PAMPS), poly(methacrylamidopropyltrimethylammonium chloride) (PMAPTAC), and an AMPS-acrylamide random copolymer $\left(\mathrm{PAMPS}_{80} \mathrm{AAm}_{20}\right)$. The critical protein charge required to induce protein-polyelectrolyte complexation, $(\mathrm{Zpr})_{\mathrm{c}}$, was found to vary linearly with the square root of the ionic strength $\left(I^{1 / 2}\right)$, i.e., with the Debye-Hückel parameter $(\kappa)$, the proportionality constant being a function of polyelectrolyte chain parameters such as intrinsic stiffness and charge density. This linearity was remarkably continuous through $\mathrm{Zpr}=0$, with $(\mathrm{Zpr})_{\mathrm{c}}$ occurring predominantly "on the wrong side" of the isoionic point; i.e., the onset of binding was typically observed when the global protein charge was of the same sign as the polyelectrolyte. Binding of BSA to the lower charge density polyanion $\left(\mathrm{PAMPS}_{80} \mathrm{AAm}_{20}\right.$ ) unexpectedly occurred under conditions where binding to the more highly charged homopolyanion (PAMPS) did not. The theoretical treatment of Muthukumar was used to interpret the linearity of $(\mathrm{Zpr})_{\mathrm{c}}$ vs $I^{1 / 2}$ and the observed influence of polyelectrolyte structural parameters. The apparent applicability of this model to the heterogeneous amphoteric protein surface suggests that binding of polyelectrolytes takes place at "charge patches" whose effective charge densities are different from, but nevertheless linearly dependent on, the global charge density.
\end{abstract}

\section{Introduction}

The intense binding to DNA of basic proteins such as histones may be the most familiar example of the interaction of proteins with an oppositely charged polyelectrolyte. In contrast, the literature on complex formation between proteins and synthetic polyelectrolytes is more diffuse, ${ }^{1}$ since it lacks the focus provided by the context of molecular biology. However, numerous studies on such complex formation and related phase separation effects have been motivated by interest in protein separations ${ }^{2}$ and enzyme stabilization and immobilization; ${ }^{3}$ it is furthermore likely that understanding and manipulation of such phenomena will play a role in protein drug deliver via microencapsulation ${ }^{4}$ and in the use of polymeric scaffolds for tissue regeneration. ${ }^{5}$

Our work on polyelectrolyte-protein complexes developed as an extension of investigations of another polyelectrolytecolloid system, namely, ionic micelles interacting with oppositely charged polymers. ${ }^{6}$ Relevant findings may be summarized as follows: (1) At any ionic strength, no interactions occur unless the micelle surface charge density exceeds some critical value $\sigma_{\mathrm{c}}$. (2) At fixed ionic strength, $\sigma_{\mathrm{c}}$ varies inversely with polymer linear charge density $\xi .^{7}$ (3) The relationship among these three variables, at least to a first approximation, is given by eq 1 , where $\kappa \sim I^{1 / 2}$ is the Debye-Hückel parameter.

$$
\sigma_{\mathrm{c}} \xi=(\mathrm{const}) \kappa
$$

This result is consistent with several theoretical treatments. ${ }^{8}$ (4) At $\sigma>\sigma_{\phi}$, bulk phase separation (either coacervation or precipitation) occurs. (5) The magnitude of $\sigma_{\phi}-\sigma_{\mathrm{c}}$ depends, in a complicated way, on inter alia stoichiometry and micelle structure, ${ }^{9}$ but within this range, soluble macromolecular complexes are formed reversibly and may be characterized by numerous scattering, hydrodynamic, and spectroscopic methods. Many of these conclusions have been shown to apply to protein-polyelectrolyte complexes as well. In particular, insight into the structure of soluble complexes has been gained from turbidimetry, ${ }^{10}$ dynamic light scattering, ${ }^{11}$ static light scattering, ${ }^{12}$ fluorescence spectroscopy, ${ }^{13}$ circular dichroism, ${ }^{14}$ electrophoretic light scattering, ${ }^{15}$ and potentiometric titration. ${ }^{16}$

Our focus in the present study is the relevance of eq 1 to polyelectrolyte-protein systems. In this case $\sigma$ depends on $\mathrm{pH}$, and complex formation with strong ( $\mathrm{pH}$-independent) polycations occurs upon addition of base. While changes in scattering intensity with $\mathrm{pH}$ appear to be more subtle than the corresponding changes observed at $\sigma_{\mathrm{c}}$ for micellar systems, a critical $\mathrm{pH}$ may be defined by electrophoretic mobility or careful turbidimetry. $\mathrm{pH}_{\mathrm{c}}$ depends in the expected way on ionic strength. However, $\mathrm{pH}_{\mathrm{c}}$ is often observed "on the wrong side" of the isoelectric point; i.e., basic proteins such as ribonuclease and lysozyme will complex with strong polycations at $\mathrm{pH}<$ $\mathrm{p} I,{ }^{15}$ and $\beta$-lactoglobulin has been observed to bind to sodium polystyrene sulfonate at $\mathrm{pH}>\mathrm{p} I .{ }^{17}$ Such observations can best be explained in terms of protein charge heterogeneity, in that "charged patches", opposite in sign to the net protein average (Zpr), can provide sites for binding to polymers with the same charge sign as Zpr. Similar effects have been observed in protein ion exchange chromatography, with proteins being retained "on the wrong side" of $\mathrm{p} I .^{18}$

Given the complexity of protein surface charge heterogeneity, it would seem unlikely that eq 1 could be directly applied to protein-polyelectrolyte complexes. Nevertheless, we recently observed a remarkable linear dependence of $(\mathrm{Zpr})_{\mathrm{c}}$ on $I^{1 / 2}$ for complex formation between bovine serum albumin (BSA) and the strong polycation poly(diallyldimethylammonium chloride) (PDADMAC) ${ }^{19}$ PDADMAC is atypical in that its charge 


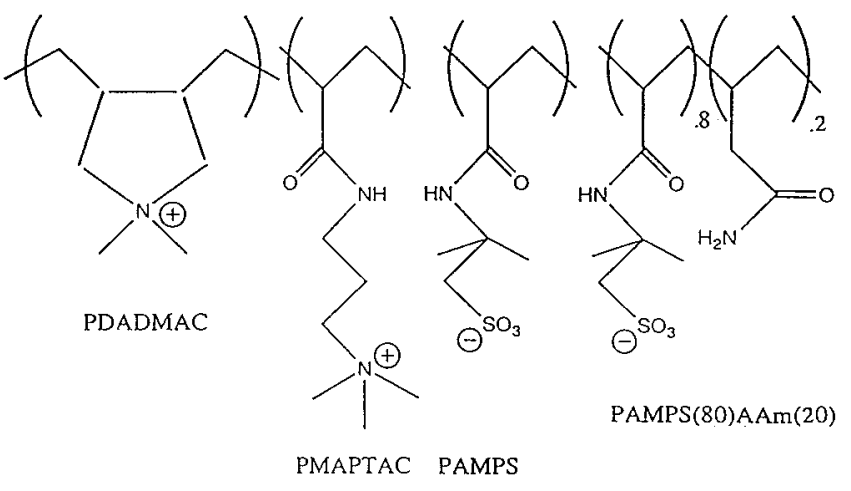

Figure 1. Molecular structures of PDADMAC, PMAPTAC, PAMPS, and PAMPS $_{80} \mathrm{AAm}_{20}$.

TABLE 1: Polyelectrolyte Chain Parameters ${ }^{26,29}$

\begin{tabular}{lllcl}
\hline $\begin{array}{c}\text { chain } \\
\text { parameter }^{a}\end{array}$ & PDADMAC & PAMPS & PAMPS(80) & PMAPTAC \\
\hline$A(\AA)$ & 6.2 & 2.5 & 3.1 & 2.5 \\
$\xi=l_{\mathrm{B}} / A$ & 1.15 & 2.84 & 2.29 & 2.84 \\
$\mathrm{lp}_{0}(\AA)$ & 27 & 24 & 24 & 24
\end{tabular}

${ }^{a} A$ is the charge spacing, $\xi$ is the linear charge density, and $l \mathrm{p}_{0}$ is the bare persistence length.

density is relatively low (ca. 6-6.5 $\AA$ between charge sites) and stiff (bare persistence length $1 p_{0}=2.7 \mathrm{~nm}$ ). In this work, we extend our studies by examining the critical conditions for complex formation with a high charge density polycation, poly(methacrylamidopropyltrimethylammonium chloride) (PMAPTAC), a high charge density polyanion, poly(acrylamidomethylpropyl sulfonate) (PAMPS), and a low charge density polyanion, the copolymer of AMPS and acrylamide (PAMPS ${ }_{80^{-}}$ $\mathrm{AAm}_{20}$ ). The results are interpreted in terms of theoretical treatments by Joanny ${ }^{20}$ and Muthukumar. ${ }^{21}$

\section{Experimental Section}

Materials. BSA ( $\mathrm{MW}=68 \mathrm{~K}$ and $\mathrm{p} I=4.9$ ) was purchased from Boehringer Mannheim as $99 \%$ pure protein and used without further purification. PDADMAC was a commercial sample of "Merquat 100", received from the Calgon Corp. with a molecular weight of $250 \mathrm{~K}$. PMAPTAC was donated by Clairol Corp. (MW $\approx 450 \mathrm{~K}$ ). PAMPS was synthesized by free radical polymerization $(\mathrm{MW} \approx 600 \mathrm{~K})$ by Don McQuigg of Reilly Industries. PAMPS ${ }_{80} \mathrm{AAm}_{20}$ was prepared by Takeshi Sato at Osaka University (MW $\approx 2000 \mathrm{~K}$ ). Polymer structures are shown in Figure 1, and relevant chain parameters are shown in Table 1.26,29 All polymer samples were purified by dialysis in nominal 1200 molecular weight cutoff tubing for 2 days and freeze-dried prior to use. $\mathrm{HCl}$ and $\mathrm{NaOH}$ solutions and $\mathrm{NaCl}$ were from Fisher and used as received.

Turbidimetric Titrations. The dependence of solution turbidity on $\mathrm{pH}$ ("type 1 titration") was obtained by the addition of either $0.1 \mathrm{M} \mathrm{NaOH}$ or $0.1 \mathrm{M} \mathrm{HCl}$ to a protein-polymer mixture at constant ionic strength and at constant polymer $(0.120$ $\mathrm{g} / \mathrm{L})$ and protein concentrations $(0.600 \mathrm{~g} / \mathrm{L})$. Protein and polymer solutions were prepared independently and filtered through Gelman $0.2 \mu \mathrm{m}$ filters prior to mixing. After titrant addition, the solution was gently stirred until a stable turbidity $( \pm 0.1 \% \mathrm{~T})$ reading was obtained. The stirring time was generally $2-3 \mathrm{~min}$, and reversibility with respect to $\mathrm{pH}$ was always observed. A nitrogen purge was employed during all titrations. Polymer-free blanks were used to eliminate the effect of free protein scattering.
An Orion $\mathrm{pH}$ meter with a combination electrode was used to monitor solution $\mathrm{pH}$. Transmittance was monitored with a Brinkman PC 800 colorimeter, connected to a $2 \mathrm{~cm}$ path length optical probe. The turbidity was reported as $100-\% \mathrm{~T}$, and $\% \mathrm{~T}$ fluctuations $( \pm 0.1 \%)$ were treated by consistently selecting the highest transmittance. Total intensity and diffusivity measurements were made with a Protein Solutions (Charlottesville, VA) DynaPro 801 dynamic light scattering instrument, which utilizes an avalanche photodiode detector and a solidstate $30 \mathrm{~mW}, 780 \mathrm{~nm}$ laser. Analysis of the autocorrelation curve by cumulants leads to an apparent average diffusion $(D)$ coefficient from which an apparent radius is calculated as $R_{\mathrm{S}}$ $=k T / 6 \pi \eta D$, where $\eta$ is the solvent viscosity, $k$ is the Boltzmann constant, and $T$ is the temperature.

Potentiometric Titrations. The charge on BSA in both the complexed and uncomplexed states was established using potentiometric titrations. Two $50 \mathrm{~mL}$ aliquots of a $0.6 \mathrm{~g} / \mathrm{L}$ BSA solution, at the desired $\mathrm{pH}$ and ionic strength, were extracted by mass and purged with $\mathrm{N}_{2}$. During the nitrogen purge, a $0.0300 \mathrm{~g}$ sample of polyelectrolyte was added to one of the aliquots ([polymer] $=0.6 \mathrm{~g} / \mathrm{L}$ ). Both aliquots were then titrated to a predetermined final $\mathrm{pH}$ using either $0.5 \mathrm{M} \mathrm{NaOH}$ or $0.5 \mathrm{M}$ $\mathrm{HCl}$. To account for the titrant volume associated with the change in $\mathrm{pH}$, a blank (polymer- and protein-free) was also titrated. For all three titrations, the initial solvent mass $( \pm 0.001$ g) and initial $\mathrm{pH}( \pm 0.005)$ were constant. Titration curves for BSA and BSA with polymer were corrected by subtraction of blank titrant volume from sample titrant volume at each $\mathrm{pH}$. The charge on BSA, relative to the charge at the initial $\mathrm{pH}$, was calculated using the blank-corrected titration curve, the mass of BSA in the solution, the molecular weight of BSA, and the concentration of the titrant. Since the charge at the isoelectric point (the $\mathrm{pH}$ of zero mobility) is ionic strength dependent, the charge was calculated relative to the isoionic point $(\mathrm{pH}=5.5)$.

Protein Surface Modeling. A 2-dimensional hypothetical representation of the BSA surface was produced in order to help visualize some of the phenomena invoked (i.e., proton mobility, charge complementarity, and multiple binding patches). Amino acid locations were determined by scaling the surface residues from the 3-dimensional model of a BSA subdomain, given in ref 24. As will be subsequently displayed in Figure 5, six of these subdomains were aligned (dashed lines) to emphasize the overall dimensions of BSA. The degree of protonation $(\alpha)$ for the charged residues was calculated using the HendersonHasselbalch expression in combination with the $\mathrm{pH}$ and the $\mathrm{p} K_{\mathrm{a}}$ values given in ref 28 . The location of the charges within each subdomain was randomized, with the restrictions that (1) the total number of charges, across the entire protein surface, is consistent with the given $\mathrm{pH}$ and the calculated $\alpha$ values and (2) the location of the charge corresponds to the location of that specific type of amino acid within the subdomain.

It is recognized that our 2-dimensional model is simplistic. However, this model allows us to place the emphasis on charge spacing and heterogeneity, while avoiding the obvious complications associated with 3-dimensional representations, such as surface roughness and curvature.

\section{Results}

Figure 2 shows the results for a typical type 1 titration of the BSA-PDADMAC system. Three regions can be defined by the changes in turbidity and $90^{\circ}$ scattering intensity observed in Figure 2. In region 1, Coulombic repulsive forces between the positively charged protein and the positively charge polyelectrolyte prohibit the formation of complexes. In region 2, 


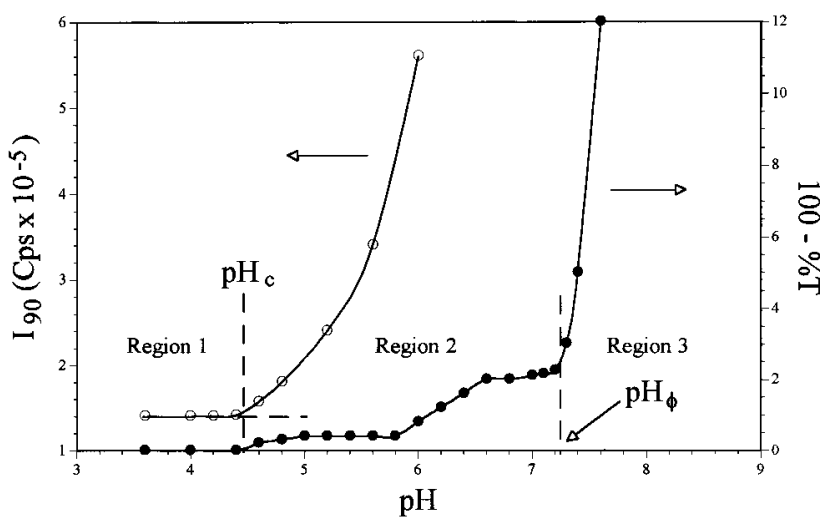

Figure 2. "Type 1 titration" for $[\mathrm{BSA}]=0.6 \mathrm{~g} / \mathrm{L}$ and $[\mathrm{PDADMAC}]$ $=0.12 \mathrm{~g} / \mathrm{L}$ in $0.1 \mathrm{M} \mathrm{NaCl}$ using $100-\% \mathrm{~T}(\bullet)$ and $90^{\circ}$ scattering intensity $(\mathrm{O})$.

designated as the primary or soluble complex region, an increase in turbidity and scattering intensity indicates that either the concentration or the molecular weight of the complex is increasing. In region 3 , the sharp increase in turbidity indicates intercomplex aggregation. These regions can be considered as separate phases, with $\mathrm{pH}_{\mathrm{c}}$ representing the boundary between the nonassociative and primary phases and $\mathrm{pH}_{\phi}$ representing the boundary between the primary and aggregate phases.

Previous studies have shown that, for a given proteinpolyelectrolyte system, $\mathrm{pH}_{\mathrm{c}}$ is independent of macromolecular concentration. ${ }^{19}$ However, the protein/polymer mass ratio $r$ does have an influence on the sensitivity of various techniques used to detect $\mathrm{pH}_{\mathrm{c}}$. At large $r$ values (excess protein), the equilibrium $\left[\mathrm{P}+\mathrm{n}(\mathrm{pr}) \leftrightarrow \mathrm{P}(\mathrm{pr})_{\mathrm{n}}\right]$ shifts in the direction of the primary complex, generally producing high molecular weight species which are readily detected using standard light scattering techniques. ${ }^{15}$ At low $r$ (excess polyelectrolyte), the concentration and possibly the molecular weight of complex are substantially decreased. Hence, changes in the concentration and molecular weight of the primary complex may not be large enough for easy detection of $\mathrm{pH}_{\mathrm{c}}$ by turbidimetric or $90^{\circ}$ intensity light scattering techniques. Under these conditions, other techniques may be better suited for detection of $\mathrm{pH}_{\mathrm{c}}$.

When proteins bind to polyelectrolytes, the proximity of the charged polymer can induce subtle changes in the $\mathrm{p} K$ values of the surface amino acids on the protein. Under conditions where the majority of the proteins are bound, i.e., excess polyelectrolyte, potentiometric titrations can be used to monitor these changes in protein $\mathrm{p} K$. Hence, potentiometric titrations are ideally suited for detection of $\mathrm{pH}_{\mathrm{c}}$ under low $r$ conditions. Figure 3a,b shows the $\mathrm{pH}$ dependence of $90^{\circ}$ scattering intensity and turbidity, along with the potentiometric titration results, for the BSA-PMAPTAC system in $0.1 \mathrm{M} \mathrm{NaCl}$. Also included in Figure $3 \mathrm{a}$ is the apparent Stokes radius from QELS. The results indicate that the measured value of $\mathrm{pH}_{\mathrm{c}}$ is independent of the technique; consequently, any subset of these four methods may be chosen on the basis of sensitivity.

The dependence of protein charge at $\mathrm{pH}_{\mathrm{c}}\left[(\mathrm{Zpr})_{\mathrm{c}}\right]$ on the ionic strength constitutes a phase boundary. Figure 4 shows the $(\mathrm{Zpr})_{\mathrm{c}}$ phase boundaries for PDADMAC, PMAPTAC, PAMPS, and $\mathrm{PAMPS}_{80} \mathrm{AAm}_{20}$. Since the focus of this study is the effect of polyelectrolyte linear charge density on $(\mathrm{Zpr})_{\mathrm{c}}$, the $(\mathrm{Zpr})_{\phi}$ phase boundaries are not included.

\section{Discussion}

The results for BSA with PDADMAC shown in Figure 4 appear at first paradoxical. The simplicity of the dependence
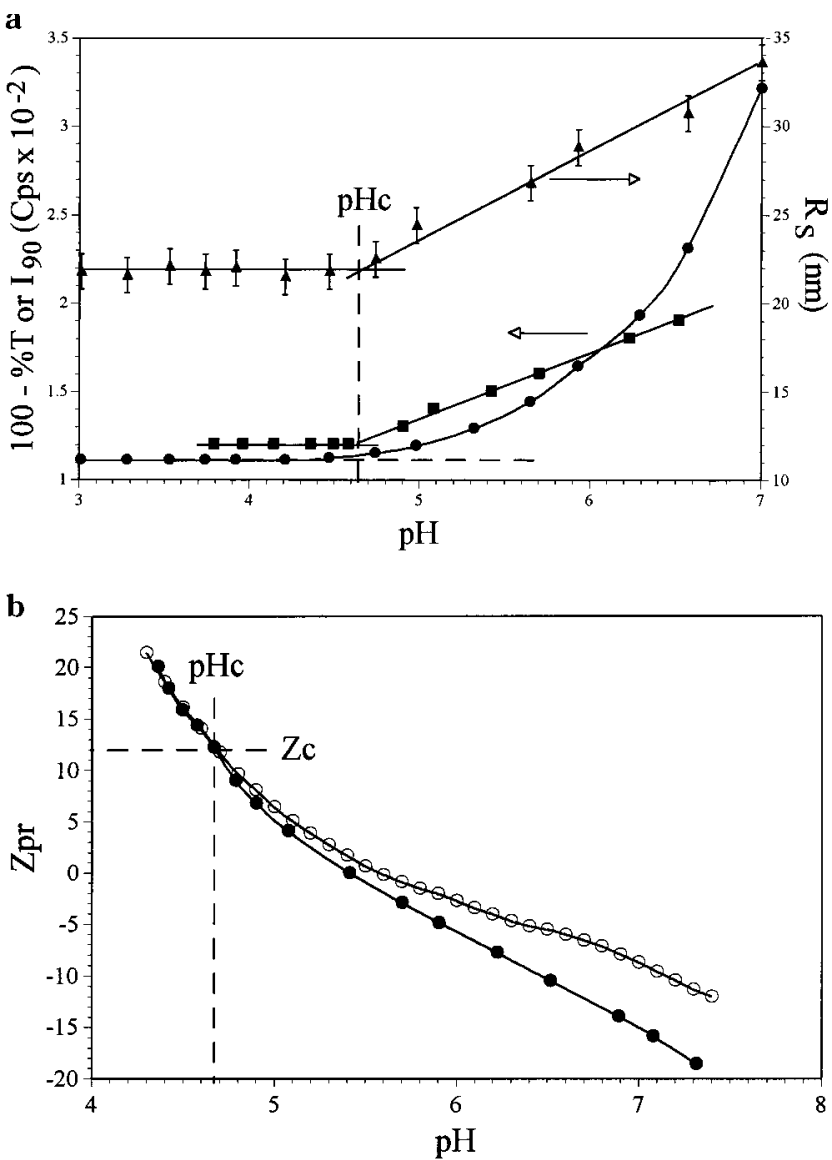

Figure 3. (a) Turbidity and light scattering results for BSAPMAPTAC at $I=0.1 \mathrm{M} \mathrm{NaCl},[\mathrm{BSA}]=0.6 \mathrm{~g} / \mathrm{L}$, and $r=5 ;(\mathbf{\Delta})$ apparent Stokes radius (right axis), (回) turbidity as $100-\% \mathrm{~T}$ (left axis), and (๑) scattering intensity as counts/s $\times 10^{-2}$ (left axis). (b) Potentiometric titrations for BSA-PMAPTAC $(\bullet)$ and BSA $(O)$ under the same conditions. $(\mathrm{Zpr})_{\mathrm{c}}$ is calculated relative to the isoionic point (5.5).

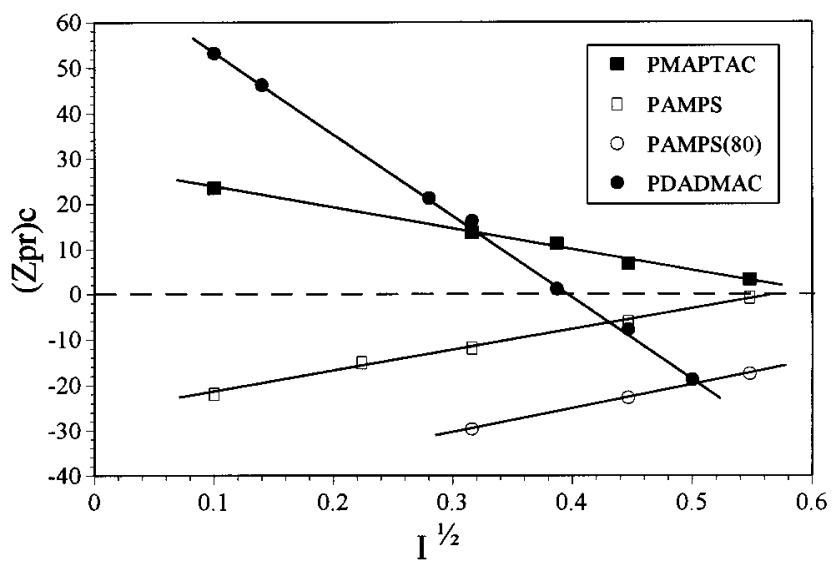

Figure 4. Ionic strength $\left(I^{1 / 2}\right)$ dependence of $(\mathrm{Zpr})_{\mathrm{c}}$ for BSA with $(\mathbf{\square})$ PMAPTAC, ( $\square$ ) PAMPS, (O) PAMPS ${ }_{80} \mathrm{AAm}_{20}$, and (๑) PDADMAC. The box in the figure lists the polyelectrolytes in order of decreasing linear charge density.

of $(\mathrm{Zpr})_{\mathrm{c}}$ on $\kappa$ strongly suggests that net protein charge governs complex formation, but complexes nevertheless form readily when $(\mathrm{Zpr})_{\mathrm{c}}$ is positive. (It might be argued that complexation produces a $\mathrm{p} K$ shift such that the effective protein isoionic $\mathrm{pH}$ may be reduced upon complex formation; titration studies show this effect to be small but measurable at $I \leqslant 0.03$, but essentially negligible at the higher ionic strengths employed in this study. ${ }^{16}$ ) Indeed, the phase boundary for PDADMAC is perfectly 
continuous through $\mathrm{Zpr}=0$. This result shows that the isoionic point has no special meaning for complex formation.

Recently, Pefferkorn ${ }^{22}$ demonstrated adsorption of polyampholytes to charged surfaces when the net polyelectrolyte charge was of the same sign as the surface (i.e., both negative). Related calculations by Joanny ${ }^{20}$ revealed that the numerous configurations available to the adsorbed polyampholyte include many favorable ones in which cationic polymer residues reside in proximity to the surface, with anionic residues at more distal positions. The enthalpy of such bound states may be strongly favorable relative to the unbound state.

In the BSA-PDADMAC case, the protein is the ampholytic "surface" which adsorbs the homopolycation. By analogy, one may easily visualize polymer configurations that favor contacts with $\mathrm{CO}_{2}^{-}$sites while avoiding contacts with $\mathrm{NH}_{3}{ }^{+}$groups. Thus, electrostatic adsorption may occur below $\mathrm{p} I$. The number of favorable configurations will of course increase upon conversion of $\mathrm{CO}_{2} \mathrm{H}$ to $\mathrm{CO}_{2}{ }^{-}$and $\mathrm{NH}_{3}{ }^{+}$to $\mathrm{NH}_{2}$, so that binding becomes more favorable with increase in $\mathrm{pH}$. Consequently, at higher $\mathrm{pH}$ binding can occur in the presence of a higher concentration of screening electrolyte, thus accounting for the increase in critical net protein charge with increase in ionic strength. Since the binding site for the polymer carries an effective surface charge density $\left(\sigma_{\text {eff }}\right)$ that is quite different from the overall protein charge, the isoionic $\mathrm{pH}$ has no particular significance vis-à-vis binding, so the continuity of the plot in Figure 4 at $\mathrm{Zpr}=0$ is not surprising. The implication that $\sigma_{\text {eff }}$ $\sim$ Zpr will be discussed below.

If protein charges were randomly distributed, one would expect symmetrical behavior for the binding of polyanions and polycations. This is exactly what we observe in Figure 4 for the two high-charge-density polyelectrolytes, PMAPTAC and PAMPS. On the other hand, comparison of PMAPTAC and PDADMAC is less simple, since the curves cross. At low ionic strength $(I)$, PDADMAC binds more strongly, but vice versa at high $I$. Put differently, if the $\mathrm{pH}$ of a solution containing BSA, PMAPTAC, and PDADMAC were adjusted upward at low $I$, complexation with PDADMAC would occur before complexation with PMAPTAC, and contrariwise at high $I$. Figure 5b,c provides a schematic explanation. At low $I, \mathrm{pH}_{\mathrm{c}}<\mathrm{p} I$, so the protein has an excess of positive charges. Nevertheless, bound configurations of PDADMAC exist in which contacts between $\mathrm{NH}_{3}{ }^{+}$and polymer cation sites are avoided because of the large spacing $(\approx 7 \AA)$ between polymer charges (Figure 5b, frames 1-5). Unfavorable like-charge contacts would be less avoidable for PMAPTAC, which has more than twice the linear charge density of PDADMAC (Figure 5b, frame 6). Consequently, PMAPTAC does not bind at large Zpr as well as PDADMAC does. As the $\mathrm{pH}$ increases, so does the number of attractive $\mathrm{CO}_{2}{ }^{-}$groups, and charge complementarity between PMAPTAC and a series of $\mathrm{CO}_{2}{ }^{-}$groups on the protein surface is eventually attained (Figure 5c, frames 1-5).

An assumption in the foregoing analyses is that both polymer and protein charges are fixed, but in fact, only the former are "quenched" whereas the latter are "annealed" (labile). Thus, protons may migrate from $\mathrm{NH}_{3}{ }^{+}$to $\mathrm{CO}_{2}{ }^{-}$in response to $\mathrm{p} K_{\mathrm{a}}$ shifts induced by polymer binding. Along with the configurational adaptability of the bound polymer, proton migration may provide an additional mechanism for favorable binding.

Comparison of PAMPS and PAMPS ${ }_{80} \mathrm{AAm}_{20}$ indicates that intrinsic chain flexibility also plays a role in the binding of proteins to polyelectrolytes. The stronger binding by the lower charge density PAMPS ${ }_{80} \mathrm{AAm}_{20}$ can be explained on the basis of charge complementarity as discussed above. However, the
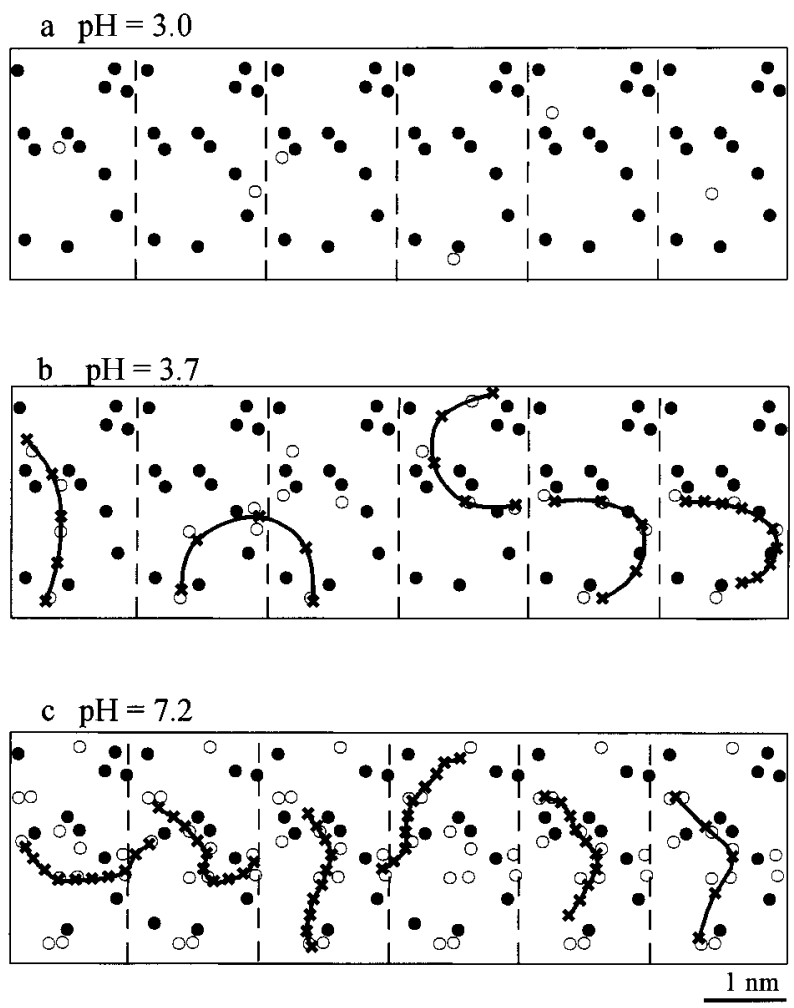

Figure 5. Schematic depiction of the surface of BSA. Amino acid charges are represented with $(O$, positive) and $(\bullet$, negative $)$, and polycation charges are depicted with $(\times)$. (a) BSA surface at low $\mathrm{pH}$ (3.0), where no polyelectrolyte binding is observed. (b) Preferential adsorption of a low charge density polycation at $\mathrm{pH}$ 3.7. (c) Preferential adsorption of a high charge density polycation at $\mathrm{pH}$ 7.2. See Experimental Section for description of model preparation.

nearly parallel behavior of the two PAMPS polyelectrolytes in Figure 4 implies that the linear charge density has only a negligible influence on the ionic strength dependence of $(\mathrm{Zpr})_{\mathrm{c}}$. This observation can be interpreted using Muthukumar's ${ }^{21}$ model for the adsorption of polyelectrolytes to charged surfaces.

In Muthukumar's model, polyelectrolyte adsorption is proposed to be a result of electrostatic interactions between a homogeneously charged planar surface and an oppositely charged polyelectrolyte. These electrostatic interactions are modulated by the surface charge density $(\sigma)$ and radius $(\Lambda)$ of the adsorbing surface and the linear charge density $(\xi)$ of the polyelectrolyte. The attenuating influence of salt on the electrostatic interactions is incorporated into the model via the DebyeHückel parameter $(\kappa)$. The attractive electrostatic contribution to polyelectrolyte adsorption is opposed by the loss of polymer conformational entropy. The Kuhn length $\left(l_{\mathrm{K}}\right)$ is representative of the polymer stiffness and is used to parametrize polyelectrolyte conformational entropy. The Bjerrum length $\left(l_{\mathrm{B}}\right)$ is a characteristic length for electrolyte solutions, and Muthukumar uses this parameter to normalize both the Kuhn length and the polyelectrolyte linear charge density. Critical conditions for polyelectrolyte adsorption are given in eq 2 , where $l_{1}$ is the renormalized Kuhn length.

$$
\left(\frac{\sigma \xi}{\kappa^{3} l_{1}}\right)\left[1-(\kappa \Lambda+1) \mathrm{e}^{-\kappa \Lambda}\right] \geqslant \frac{1}{12 \pi l_{\mathrm{B}}}
$$

For high molecular weight polyelectrolytes, the radius of gyration is much larger than the screening length $(1 / \kappa)$, and $l_{1}$ is proportional to both the bare Kuhn length $\left(l_{0}\right)$ and $\kappa$. This 


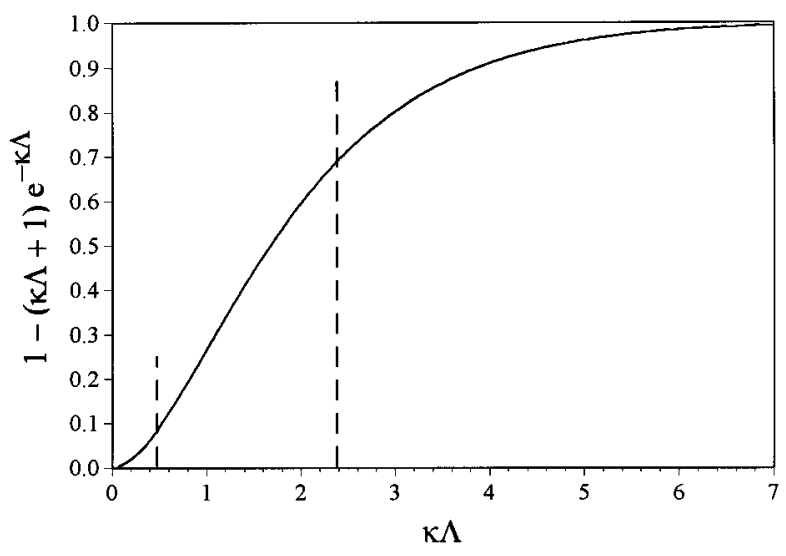

Figure 6. Plot of $\left[1-(\kappa \Lambda+1) \mathrm{e}^{-\kappa \Lambda}\right]$ as a function of $\kappa \Lambda$. The dashed lines represent the range of values applicable to the BSA study, assuming a $\Lambda$ value of ca. $1.5 \mathrm{~nm}$, with $\kappa=3.29 I^{1 / 2}\left(\mathrm{~nm}^{-1}\right)$.

proportionality is shown in eq 3 , where $\omega_{\mathrm{c}}$ is the electrostatic interaction parameter $\left(\omega_{\mathrm{c}}=l_{\mathrm{B}} / A^{2}\right), N$ is the number of Kuhn segments in the contour length, and $A\left(=l_{\mathrm{B}} / \xi\right)$ is the contour distance between charges on the polyelectrolyte: ${ }^{21,23}$

$$
l_{1} \sim\left(\frac{\omega_{\mathrm{c}}}{l_{0} \kappa^{2}}\right)^{0.4} N^{0.2} l_{0}=\frac{N^{0.2} l_{0}^{0.6}}{A^{0.8} \kappa^{0.8}}
$$

As shown in Figure 6, the second term in eq $2\{1-(\kappa \Lambda+$ 1) $\left.\mathrm{e}^{-\kappa \Lambda}\right\}$ is linear with $\kappa \Lambda$ in the range of small $\kappa \Lambda$ values. After the appropriate substitutions, the preceding expressions reduce to the form shown in eq 4, which predicts that the intrinsic chain stiffness $\left(l_{0}\right)$ has a larger effect on $\sigma_{\mathrm{c}}$ than the linear charge density.

$$
\sigma_{\mathrm{c}} \sim\left(\frac{l_{0}^{0.6} N^{0.2}}{\xi^{0.2} \Lambda}\right) \kappa^{1.2}
$$

Therefore, despite the difference in $\xi$, the slopes of the phase boundaries $\left(\partial \sigma_{\mathrm{c}} / \partial \kappa\right)$ for PAMPS and PAMPS ${ }_{80} \mathrm{AAm}_{20}$, two polyelectrolytes with similar bare persistence lengths, are nearly parallel, with the small difference in slope arising from the difference in $\xi^{1 / 5}$. Since $(\mathrm{Zpr})_{\mathrm{c}}<0$ for PAMPS and $\mathrm{PAMPS}_{80^{-}}$ $\mathrm{AAm}_{20}$, the vertical shift toward stronger binding for PAMPS P0- $^{-}$ $\mathrm{AAm}_{20}$ is likely to be a result of same sign repulsive interactions, a factor not included in Muthukumar's model. For the cationic polyelectrolytes, PDADMAC (I) and PMAPTAC (II) where $l_{\mathrm{I}}$ $>l_{\mathrm{II}}$ and $\xi_{\mathrm{I}} \ll \xi_{\mathrm{II}}$, eq 4 correctly predicts that the slope of the phase boundary will be steeper for PDADMAC than for PMAPTAC. With regard to the $\kappa$ dependence in eq 4 , Figure 7 shows the data from Figure 4 plotted vs $I^{0.6}$. As seen, it is not possible to distinguish $\kappa$ dependence and $\kappa^{1.2}$ dependence.

Since $\Lambda$ is representative of the number of protein charges interacting with the polyelectrolyte, the presence of $\Lambda$ in eq 4 is not unexpected. However, the linearity of $\sigma_{\mathrm{c}}$ with $\kappa^{1.2}$ taken together with eq 4 implies that $\Lambda$ is constant (independent of either $\mathrm{pH}$ or ionic strength). Because of the large loss in polyelectrolyte conformational entropy upon binding, it is unlikely that a single contact point releases enough enthalpic energy to stabilize the binding event. It is more reasonable to assume multiple links involving contiguous binding sites. The number and the relative location of these binding sites would be dictated by the distribution of charges on the polyelectrolyte and the chain stiffness. Because of the proximity of protein and bound polymer groups, the effect of the bulk ionic strength

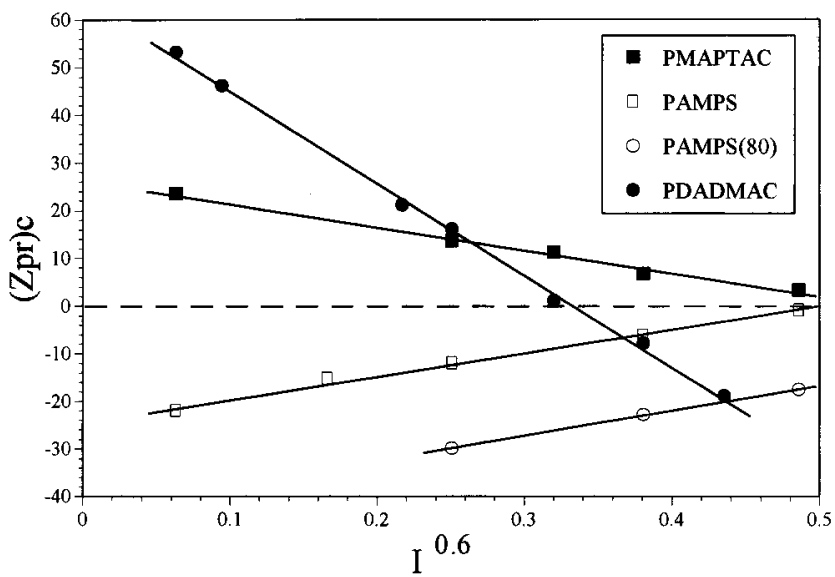

Figure 7. Ionic strength $\left(I^{0.6}\right)$ dependence of $(\mathrm{Zpr})_{\mathrm{c}}$ for BSA with $(\mathbf{\square})$ PMAPTAC, ( $\square$ ) PAMPS, (O) PAMPS ${ }_{80} \mathrm{AAm}_{20}$, and (-) PDADMAC. The box in the figure lists the polyelectrolytes in order of decreasing linear charge density.

on the persistence length can be neglected. Hence, it is the bare persistence length or the intrinsic chain stiffness which determines the size of the binding patch, resulting in a relatively constant $\Lambda$ for a given polyelectrolyte.

The presence of multiple contact points can be inferred from Muthukumar's computer simulation studies of polyelectrolyte adsorption to charged surfaces. ${ }^{21}$ These simulations showed that polyelectrolyte adsorption was initiated at a single contact point. As evident by the short lifetime, the initial polymer conformation was unstable but could be stabilized by the inclusion of neighboring contact points. Studies of complexes of strong polyanions (e.g., PAMPS) with spherical (4 nm diameter) cationic micelles provide circumstantial evidence that the bound state involves ca. $5-10$ polymer residues per micelle. ${ }^{25}$ It may also be noted that 5-10 PAMPS residues corresponds to a contour distance of $1.3-2.7 \mathrm{~nm}$, on the order of the bare persistence length $\left(1 p_{0}=2.4 \mathrm{~nm}\right){ }^{26}$ We can then suggest that the "charge patch" is actually an array of charges on the protein surface which are complementary to the distribution of charges on the polyelectrolyte binding segment and that the length of the binding segment, and hence the size of the patch $(\Lambda)$, is controlled by the intrinsic stiffness of the polymer chain. As $\mathrm{lp}_{0}$ increases, we can expect a corresponding increase in $\Lambda$.

The inference that $\partial \sigma_{\mathrm{c}} / \partial \kappa \sim \partial(\mathrm{Zpr})_{\mathrm{c}} / \partial \kappa$ implies that $\sigma_{\text {eff }} \sim$ Zpr, which might appear to conflict with the fact that the initial binding event at $\mathrm{pH}_{\mathrm{c}}$ typically occurs on the wrong side of $\mathrm{pI}$, meaning that $\sigma_{\text {eff }}$ and $\mathrm{Zpr}$ are of opposite sign. Since protein charges are not in general randomly distributed, global charge homogeneity seems unlikely. A more reasonable explanation is that there are multiple patches of similar energy on the protein surface, with each patch adding an entropic stability to the binding event. If the number of patches is large, we could envision a bound polyelectrolyte segment that has a large degree of mobility on the protein surface. Because of this mobility, the effective surface charge density would be a weighted average of all the patches, and we would expect this average to be proportional to the global charge. Hence the apparent proportionality of $\sigma_{\text {eff }}$ with $\mathrm{Zpr}$.

If multiple patches are present on the protein surface, there may be numerous polyelectrolyte conformations of similar binding affinity. These conformations in equilibrium could be described as "loose" binding. Direct evidence of this type of binding was observed by Sato et al. for lysozyme complexed with PAMPS. ${ }^{27}$ From fluorescence anisotropy measurements, the rotational lifetime of unbound lysozyme was found to be 
$1.3 \mathrm{~ns}$. When polyelectrolyte was added to the system, the rotational lifetime of lysozyme increased by $40 \%$ to $1.8 \mathrm{~ns}$. While this increase is indicative of complex formation, it is of insufficient magnitude to support a site-specific, "lock and key" binding mechanism. Multiple patches would also be consistent with the theoretical treatments presented by Muthukumar ${ }^{21}$ and Pefferkorn. ${ }^{22}$ In Muthukumar's computer simulations of polyelectrolyte adsorption to charged surfaces, numerous low-energy conformations of the adsorbed polyelectrolyte chain were observed. Pefferkorn et al. attribute the reversibility of polyampholyte adsorption to the inherent mobility or conformational freedom of the bound polyampholyte at the adsorbing surface; i.e., desorption is prohibited only when conformational freedom is lost.

The presence of multiple patches of similar binding affinity is portrayed in Figure 5. Heavy lines in Figure 5b,c represent polycations of different linear charge density and persistence length. The charge (X) spacing and the total length of the absorbed polycation segments are consistent with the linear charge densities and the bare persistence lengths of PDADMAC and PMAPTAC. Frames 1-4 in Figure 5b,c depict multiple binding patches and proton mobility, using the polycation preferentially absorbed at each $\mathrm{pH}$. In the last two frames the two polycations are compared to show the restrictions imposed by charge complementarity at low $\mathrm{pH}$ and the decrease in these restrictions at higher $\mathrm{pH}$, i.e., the preferential adsorption of low charge density PDADMAC at low $\mathrm{pH}$, contrasted to the adsorption of high charge density PMAPTAC at high $\mathrm{pH}$.

Figure 5a corresponds to $\mathrm{pH}=3.0$, where most of the carboxylate groups are protonated $(\mathrm{p} K=4.0)$, so there are not enough negative charges to constitute a binding patch, and polycation adsorption is prohibited. At $\mathrm{pH}=3.7\left(\mathrm{pH}_{\mathrm{c}}\right.$ for PDADMAC in $0.04 \mathrm{M} \mathrm{NaCl}$ ), a sufficient number of carboxylate groups have been ionized to induce PDADMAC adsorption, represented schematically in the first four frames of Figure $5 \mathrm{~b}$. The specific set of acid residues that are ionized is variable, and subtle $\mathrm{p} K$ shifts induced by the polycation could multiply the number of possible binding sites. Figure $5 \mathrm{c}$ represents the protein surface at moderate $\mathrm{pH}$ (7.2) and ionic strength (0.2). The added salt screens attractive interactions, and PDADMAC adsorption is prohibited until the surface charge density has increased enough to balance the reduction in $\kappa^{-1}$ $\left(\mathrm{pH}_{\mathrm{c}}=7.2\right)$. At $\mathrm{pH} 7.2$, effectively all of the carboxyl groups are ionized, and $\sigma_{\text {eff }}$ is large. However, this increase in $\sigma_{\text {eff }}$ reduces the importance of charge complementarity and produces patches that are more conducive to adsorption of high charge density polyelectrolytes (PMAPTAC). PMAPTAC adsorption, shown in the first five frames of Figure 5c, is characterized by a smaller $\Lambda$, arising from the larger intrinsic flexibility of PMAPTAC relative to PDADMAC, and a decrease in the total number of positive charges, arising from the deprotonation of $70 \%$ of the imidazole (His) groups. The result is preferential adsorption of the more highly charged and flexible PMAPTAC in the high ionic strength regime.

Finally, we address the question of the phase-transition-like behavior implied by " $\mathrm{pH}_{\mathrm{c}}$ ". The fact that $\mathrm{pH}_{\mathrm{c}}$ is independent of polyelectrolyte $\mathrm{MW}^{19}$ in the MW range of 104-106 implies that we need only consider interactions on the length scale of a protein molecule, i.e., $<10 \mathrm{~nm}$, the longest dimension of BSA. $^{24}$ Since PDADMAC is a relatively stiff polymer, the notion of PDADMAC "wrapping around" BSA is unrealistic, but a more reasonable deformation of the polymer chain can still give rise to numerous contacts. The first or primary contact between one polymer repeat unit and the protein results in a large loss in polyelectrolyte conformational entropy. However, since the brunt of the entropy penalty is absorbed by this primary contact, subsequent contacts with other repeat units are more favorable. This cooperativity is responsible for the abrupt appearance of a bound state. Therefore, the simultaneous binding of multiple contiguous polymer repeat units accounts for the phase-transition-like behavior observed at $\mathrm{pH}_{\mathrm{c}}$.

\section{Conclusion}

The surface charge distributions of proteins correspond to a complex pattern that changes strongly with $\mathrm{pH}$. Precise complementarity of protein charges with the charges on synthetic polyelectrolytes is highly unlikely. Nevertheless, the range of conformational arrangements for the adsorbed polyelectrolyte and the variety of possible binding sites on the protein offer some states of low energy even when the global charge suggests strong repulsion.

We observed that the critical protein charge for BSA-polyelectrolyte complex formation $(\mathrm{Zpr})_{\mathrm{c}}$ depends, to a first approximation, on $\kappa$. This observation is consistent with numerous theoretical treatments and has also been observed for micellepolyelectrolyte and dendrimer-polyelectrolyte systems. ${ }^{25}$ Contrary to micelles and dendrimers, however, the amphoteric nature of proteins introduces a charge complementarity factor that may oppose the expected increase in binding with increasing polyelectrolyte charge density. Theories for the adsorption of polyelectrolytes to charged surfaces ${ }^{21}$ indicate that the effect of intrinsic polyelectrolyte stiffness on $(\mathrm{Zpr})_{\mathrm{c}}$ exceeds the effect of polyelectrolyte linear charge density. This prediction is supported by the results for both the polyanionic and polycationic systems.

Acknowledgment. This research was supported by National Science Foundation Grant DMR-9619772. The authors would like to thank M. Muthukumar and F. von Goeler for their assistance.

\section{References and Notes}

(1) Xia, J.; Dubin, P. L. In Macromolecular Complexes in Chemistry and Biology; Dubin, P. L., Bock, J., Davis, R. M., Schulz, D., Thies, C., Eds.; Springer-Verlag: Berlin, 1994; Chapter 15.

(2) Dubin, P. L.; Gao, J.; Mattison, K. Sep. Purif. Methods 1994, 23,1 .

(3) Kokufuta, E. Prog. Polym. Sci. 1992, 16, 1.

(4) Burgess, D. In Macromolecular Complexes in Chemistry and Biology; Dubin, P. L., Bock, J., Davis, R. M., Schulz, D., Thies, C., Eds.; Springer-Verlag: Berlin, 1994; Chapter 17.

(5) Chang, T. M. S. Biotechnol. Annu. Rev. 1995, 1, 267.

(6) Dubin, P. L.; Th, S. S.; McQuigg, D. W.; Chew, C. H.; Gan, L. M. Langmuir 1989, 5, 89.

(7) Dubin, P. L.; Curran, M. E.; Hua, J. Langmuir 1990, 6, 707.

(8) (a) Odijk, T. Langmuir 1991, 7, 1992. (b) Manning, G.; et al. Paper presented at Colloid and Surface Science Symposium, Stanford University, June 1994. (c) Evers, O. A.; Fleer, G. J.; Scheutjens, J. M. H. M.; Lyklema, J. J. Colloid Interface Sci. 1986, 111, 446.

(9) Xia, J.; Zhang, H.; Rigsbee, D. R.; Dubin, P. L.; Shaikh, T. Macromolecules 1993, 26, 2759.

(10) Park, J. M.; Muhoberac, B. B.; Dubin, P. L.; Xia, J. Macromolecules 1992, 25, 290.

(11) Dubin, P. L.; Murrell, J. M. Macromolecules 1988, 21, 2291.

(12) Tsuboi, A.; Tsuyoshi, I.; Hirata, M.; Xia, J.; Dubin, P. L.; Kokufuta, E. Langmuir 1996, 12, 6295 .

(13) Izumi, T.; Hirata, M.; Kokufuta, E.; Cha, H.-J.; Frank, C. W. J. Macromol. Sci., Pure Appl. Chem. 1994, A31, 39.

(14) Santucci, R.; Amiconi, G.; Ascoli, F.; Brunori, M. Biochem. J. 1986, $240,613$.

(15) (a) Xia, J.; Dubin, P. L.; Kim, Y.; Muhoberac, B. B.; Klimkowski, V. J. J. Phys. Chem. 1993, 97, 4528. (b) Li, Y.; Mattison, K.; Dubin, P. L.; Havel, H.; Edwards, S. Biopolymers 1996, 38, 527.

(16) Wen, Y.-P.; Dubin, P. L. Macromolecules 1997, 30, 7856. 
(17) Gao, J. Y.; Dubin, P. L.; Muhoberac, B. B. Anal. Chem. 1997, 69, 2945.

(18) Lesins, V.; Ruckenstein, E. Colloid Polym. Sci. 1988, 266, 1187. (19) Mattison, K. W.; Brittain, I. J.; Dubin, P. L. Biotechnol. Prog. 1995, 11,632 .

(20) Joanny, J.-F. J. Phys. II 1994, 4, 1281.

(21) (a) Muthukumar, M. J. Chem. Phys. 1987, 86, 7230. (b) Muthukumar, M. J. Chem. Phys. 1995, 103, 4723.

(22) (a) Neyret, S.; Ouali, L.; Candau, F.; Pefferkorn, E. J. Colloid Interface Sci. 1995, 176, 86. (b) Huguenard, C.; Widmaier, J.; Elaissari, A.; Pefferkorn, E. Macromolecules 1997, 30, 1434.
(23) Yamakawa, H. Modern Theory of Polymer Solutions; Harper \& Row: New York, 1971.

(24) Brown, J. R.; Shockley, P In Lipid-Protein Interactions; Jost, P. C., Griffith, O. H., Eds.; Wiley: New York, 1982; Vol. 1, Chapter 2.

(25) McQuigg, D. W. M.S. Thesis, Purdue University, 1989.

(26) Tricot, M. Macromolecules 1984, 17, 1698

(27) Sato, T.; Mattison, K. W. Unpublished results.

(28) Tanford, C.; Swanson, S. A.; Shore, W. S. J. Am. Chem. Soc. 1955, $77,6414$.

(29) Dautzenberg, H. Personal comunication. 